EPJ Web of Conferences 45, 01013 (2013)

DOI: 10.1051 /epjconf/20134501013

(C) Owned by the authors, published by EDP Sciences, 2013

\title{
On Application of EARSM Turbulence Model for Simulation of Flow Field behind Rack Station
}

\author{
V.Běták ${ }^{1 a}$, P.Sváček ${ }^{1}$, J.Novotný ${ }^{2}$, J. Fürst ${ }^{1}$ and J. Forrt ${ }^{1}$ \\ ${ }^{1}$ CTU Prague, Faculty of Mechanical Engineering, Department of Technical Mathematics, Prague, Czech Republic \\ ${ }^{2}$ CTU Prague, Faculty of Mechanical Engineering, Department of Fluid Mechanics and Thermodynamics, Prague, Czech \\ Republic
}

\begin{abstract}
This paper is interested in the mathematical modeling and approximation of the turbulent flow with strong anisotropy for the case of flow in a rack station. Turbulent models based on Boussinesq eddy viscosity assumption are traditionally employed for the solution of these types of flows. Linear eddy viscosity model and the assumption of isotropic Reynolds stress tensor are typically used in many cases. This leads to certain limitations in the cases of either separated flow or flow with significant curvature of the mean flow. The application of full Reynolds stress model is suitable for this case but it introduces at least seven additional equations and the model has much higher demand for computing time, grid parameters and discretization schemes. Therefore the Explicit Algebraic Reynolds Stress Model (EARSM) is used here. This model is based on two equations $\mathrm{k}$ - omega turbulence model with the nonlinear eddy viscosity model and explicit terms for anisotropic parts of the Reynolds stress tensor. The computational requirements are similar to the standard $\mathrm{k}$ omega model. The numerical and experimental results from PIV measurement are compared.
\end{abstract}

\section{Introduction}

CFD methods are recently used in many applications e.g. [9]. Number of them are connected with turbulent flow field that is not steady state. There are three approaches how to solve it. The first one is Direct Numerical Simulations (DNS) see e.g. [1] that hardware requirements are too high. The second one is Large Eddy Simulation (LES) see e.g. [2]. Both of these approaches leads to transient simulations which allows a detailed study of flow field topology. The most popular is Reynolds Average Navier-Stokes (RANS) see e.g. [3] approach based on modeling of the time average or mean fluid quantities. These models used Boussinesq assumption which implies isotropy in Reynolds Stresses. Reynolds stresses are modeled as

$$
-\overline{u_{i}{ }^{\prime} u_{j}{ }^{\prime}}=v_{t}\left(\frac{\partial u_{i}}{\partial x_{j}}+\frac{\partial u_{j}}{\partial x_{i}}\right)-\frac{2}{3} k \delta_{i j},
$$

This approach has some limitations, especially its applications in the regions with significant velocity gradients is questionable. Therefore several authors e.g.
$[4,5,6]$ use an additional anisotropy term in the model of Reynolds stresses approximation.

This paper deals with application of two-equation turbulence model with the anisotropy term to the approximation of the flow field nearby the PC rack station. A strong anisotropy generated by the CPU cooler in flow field is expected behind the rack blade. Moreover an interaction between jets from different blades and rack station is expected. The numerical results obtained by Explicit Algebraic Reynolds Stress Model (EARSM) $[5,6]$ turbulence model are compared with experimental data obtained by Particle Image Velocimetry (PIV) measurement and the isotropy turbulence model such as realizable $k-\epsilon \quad$ [8] or $k-\omega$ SST [7] models.

a betakv@seznam.cz 


\section{Governing equations}

Incompressible viscous turbulent flow in rack station is modeled by Reynolds Average Navier-Stokes system of equation in $\Omega \subset \mathbb{R}^{3}$ described by following system of equations

$$
\begin{gathered}
\frac{\partial}{\partial x_{j}}\left(u_{j}\right)=0, \\
\frac{\partial}{\partial x_{j}}\left(u_{i} u_{j}\right)=-\frac{\partial \tilde{p}}{\partial x_{i}}+\frac{\partial}{\partial x_{j}}\left[v_{e f f} \frac{\partial u_{i}}{\partial x_{j}}-k\left(\frac{2}{3} \delta_{i j}+a_{i j}^{(e x)}\right)\right]
\end{gathered}
$$

where $u_{i}$ are the components of the mean velocity vector, $\tilde{p}$ is the mean kinematic pressure, $v_{e f f}=v+v_{t}$ is the sum of the kinematic and the turbulent viscosity, $k$ represents the turbulent kinetic energy and $a_{i j}^{(e x)}$ is the anisotropic stress tensor.

Because the presence of strong gradients in the velocity flow field is expected, the EARSM[6] developed by the Hellsten model was used. This model is based on the twoequation $k-\omega S S T$

$$
\begin{gathered}
\frac{\partial k u_{j}}{\partial x_{j}}=P_{k}-\beta^{*} k \omega+\frac{\partial}{\partial x_{j}}\left[\left(v+v_{t} \sigma_{k}\right) \frac{\partial k}{\partial x_{j}}\right], \\
\frac{\partial \omega u_{j}}{\partial x_{j}}=\gamma \frac{\omega}{k} P_{k}-\beta \omega^{2}+\frac{\partial}{\partial x_{j}}\left[\left(v+v_{t} \sigma_{\omega}\right) \frac{\partial \omega}{\partial x_{j}}\right] \\
+\frac{\sigma_{d}}{\omega} \max \left(\frac{\partial k}{\partial x_{j}} \frac{\partial \omega}{\partial x_{j}}, 0\right) \\
, \\
+\beta_{6}\left(S_{i k} \Omega_{k l} \Omega_{l j}+\Omega_{i k} \Omega_{k l} S_{l j}-I I_{\Omega} S_{i j}-\frac{2}{3} I V \delta_{i j}\right) \\
+\beta_{9}\left(\Omega_{i k} S_{k l} \Omega_{l m} \Omega_{m j}+\Omega_{i k} \Omega_{k l} S_{l m} \Omega_{m j}\right)
\end{gathered}
$$

where $\omega$ is the rate of specific dissipation, $S$ represent the strain rate tensor and $\Omega$ is the vorticity tensor which contain the extra vorticity part

$$
\Omega_{i j}=\frac{1}{2} \tau\left(\frac{\partial u_{i}}{\partial x_{j}}-\frac{\partial u_{j}}{\partial x_{i}}\right)-\frac{\tau}{A_{0}} \Omega_{i j}^{(r)},
$$

where

$$
\Omega_{i j}^{(r)}=-e_{i j k} \frac{I I_{S}^{2} \delta_{k m}+12 I I I_{S} S_{k m}+6 I I_{S} S_{k l} S_{l m}}{2 I I_{S}^{3}-12 I I I_{S}^{2}} S_{p r} \dot{S}_{r q} e_{p q m}
$$

The $\dot{S}$ is a time derivation of strain rate tensor, $I I_{S}, I I I_{S}, I I_{\Omega}, I V$ are the invariants of the strain rate and vorticity tensors (see [6] ).

The timescale $\tau$ is defined as

$$
\tau=\max \left(\frac{1}{\beta^{*} \omega}, 6.0 \sqrt{\frac{v}{\beta^{*} k \omega}}\right) .
$$

For more details about model see [6].

\section{Testing case description}

The simplified model of the rack station and computational domain is shown in Figure 1. The rack station is enclosed in the bounded computational domain with the dimensions are $1500 \times 300 \times 1100 \mathrm{~mm}$. Several geometrical details of the real geometry were neglected. Symmetrical flow field in the rack station is assumed.

There are three inlets located approximately in the middle of the rack station $(1055 \times 600 \times 960 \mathrm{~mm})$. On inlets the fixed flow rate of $0.0305 \mathrm{~m}^{3} \mathrm{~s}^{-1}$ is prescribed and the turbulent intensity at $5 \%$ with mixing length equal to 5 $\mathrm{mm}$ is assumed.

Two meshes consisting of hexahedral and polyhedral cells are used. The coarse mesh used for $k-\epsilon$ or $k-\omega$ SST contains approximately $2 \times 10^{6}$ of cells and fine mesh (EARSM) contains approximately $7 \times 10^{6}$ of cells.

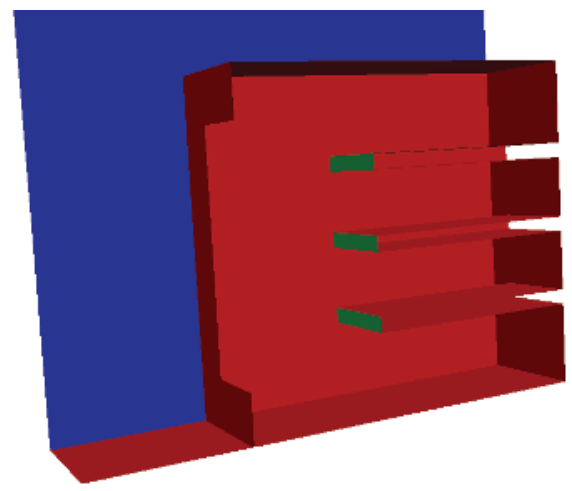

Fig. 1. Testing case geometry ( red - wall bc, green inlet bc, blue outlet bc ). 


\section{Results}

The numerical results are compared with PIV measurement in a plane which is located in the distance of $65 \mathrm{~mm}$ parallel to symmetry plane. The x component of velocity vectors are compared in the selected distance from the rack station.

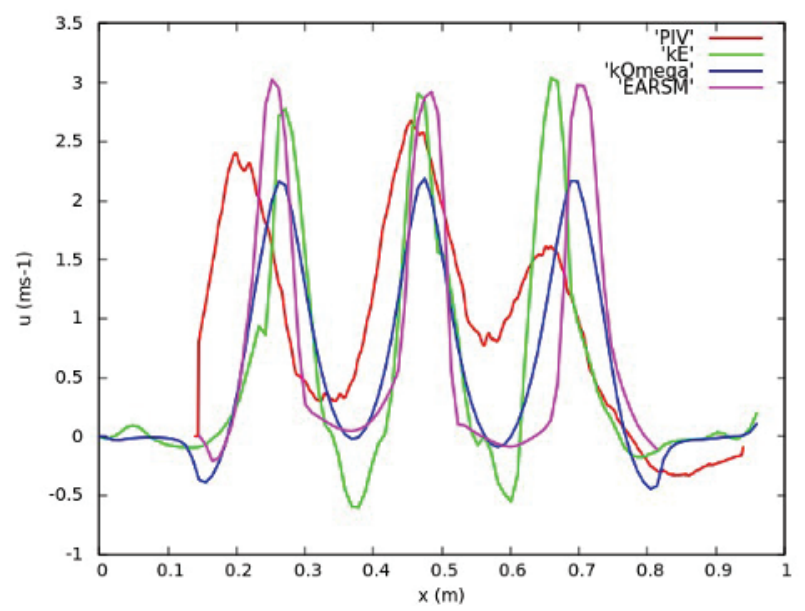

Fig. 2. Velocity profiles in distance $100 \mathrm{~mm}$

Figure 2 shows the comparison in distance $100 \mathrm{~mm}$. The numerical simulations give the position and magnitude of side jets differently. Lower magnitude of velocities predicted by $k-\omega S S T$ are observed.

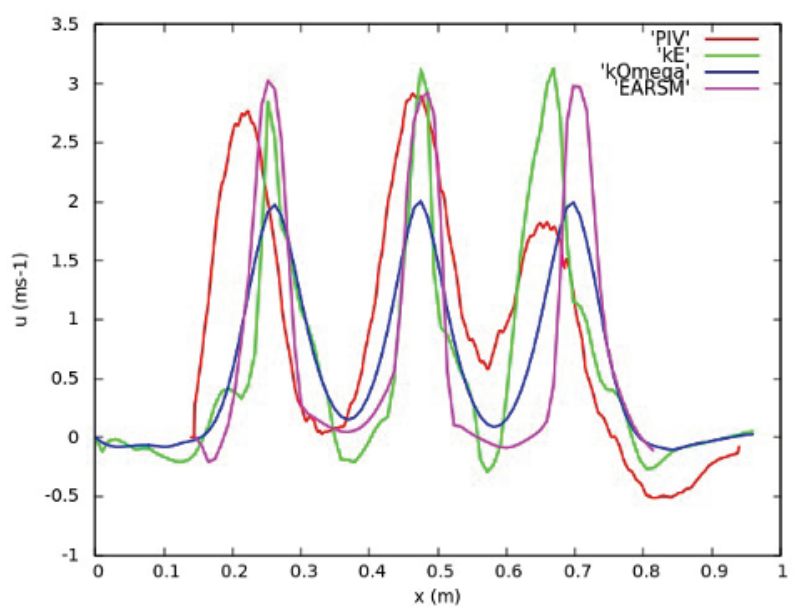

Fig. 3. Velocity profiles in distance $500 \mathrm{~mm}$

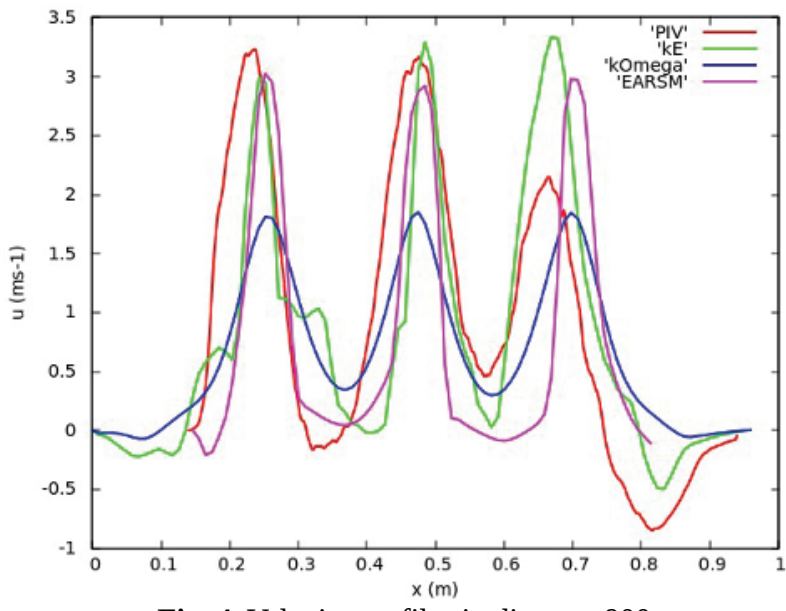

Fig. 4. Velocity profiles in distance $900 \mathrm{~mm}$

Figure 3 shows comparison in distance $500 \mathrm{~mm}$ and Figure 4 shows comparison in distance $900 \mathrm{~mm}$. Similar as in Figure 1 lower magnitude of velocities predicted by $k-\omega S S T$ are observed.

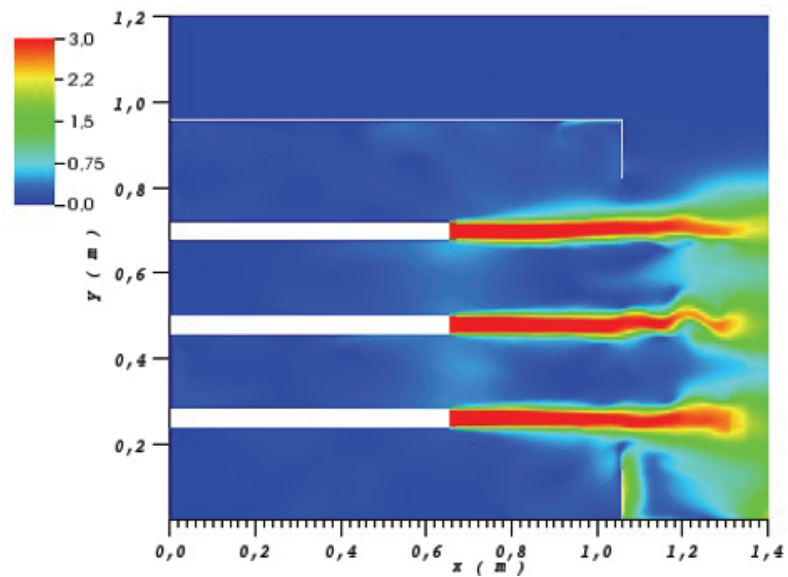

Fig. 5. Detail of velocity magnitude in measurement plane 


\section{Conclusion}

The EARSM and realizable $k-\epsilon$ turbulence models produced similar results that are comparable with the experimental data obtained for middle jets obtained by PIV. Results for the side jets seem to be affected by the low resolution of both meshes close the window in the backside of the rack. The agreement of the $k-\omega S S T$ results with the PIV decreases with the increasing distance from rack station.

The future work will be focused on improvement quality of the numerical results. This will be done by detailed modeling flow inside rack blades and the mesh refinement in the near of sharp edges.

\section{Acknowledgment}

This work was supported by the Grant Agency of the Czech Technical University in Prague, grant No. SGS 10/243/0HK2/3T/12 and by Technology Agency of Czech Republic, grant No. TA01010184 .

\section{References}

1. T. R. Bewley , P. Moin, R. Teman, J. Fl. Mech. 447 (2001)

2. J. A. Langford and R. D. Moser, J. Fl. Mech. 398

(1999)

3. P.R. Spalart: Int. J. H. Fl. Fl. 21, 3 (2000)

4. P. A. Durbin, AIAA Journal 33,4 (1995)

5. S. W. Johansson, Int. J. H. Fl. Fl. 23 (2002)

6. A. Hellsten, Helsinki University of Technology (2004)

7. R.F.Menter, 24th AIAA Fluid Dynamics Conference (1993)

8. T.H. Shih, W.W. Liou, A. Shabbir, Z. Yang, NASA

STI/Recon (1994)

9. J. Halama, J. Dobeš, J. Fořt, J. Fürst, K. Kozel,

J. Comp. App. Math. 234, 7 (2010) 